\title{
ABC Management Research on Staff in Newly-built Undergraduate Colleges \\ Jiming $\mathrm{Chu}^{1}$ and Jin $\mathrm{Wu}^{2}$ \\ ${ }^{1}$ Library of Xuzhou University of Technology, Xuzhou, Jiangsu 221018; \\ ${ }^{2}$ School of Management of Xuzhou University of Technology, Xuzhou, Jiangsu 221018
}

\begin{abstract}
On the basis of analyzing the current status of the library staff in the newly-built undergraduate colleges, this paper discusses the necessity of newly-built undergraduate colleges to developing $\mathrm{ABC}$ management in following four aspects: improving the quality of the staff, completing the construction and conversion of staff grade; realizing the scientific staffing and further optimizing the quality evaluation. It also points out that the staff in $\mathrm{A}, \mathrm{B}, \mathrm{C}$ grades shall adopt different kinds of managements as authorized management, participatory management and instructional management. In addition, it introduces the procedures of constructing the ABC management of newly-built undergraduate colleges' library.
\end{abstract}

Keywords: Newly-Built undergraduate college; Library; Staff; ABC management

\section{新建本科院校图书馆职工 $\mathrm{ABC}$ 管理研究}

\author{
储济明 ${ }^{1}$ ，吴瑾 ${ }^{2}$
}

（1.徐州工程学院图书馆，江苏 徐州 221018；2.徐州工程学院管理学院，江苏 徐州 221008）

摘要: 在分析新建本科院校图书馆职工现状的基础上, 从提升职工素质、完善职工等级建设和转换、实现职工配置的科 学化、进一步优化素质评测四方面论述了新建本科院校图书馆开展职工 $\mathrm{ABC}$ 管理的必要性, 指出对 $\mathrm{A} 、 \mathrm{~B} 、 \mathrm{C}$ 类职工应分 别采用授权式管理、参与式管理和指示式管理, 介绍了新建本科院校图书馆实施职工 $\mathrm{ABC}$ 管理的步骤。

关键词: 新建本科院校; 图书馆; 职工; $\mathrm{ABC}$ 管理

中图分类号: G215 文献标识码: A

引言

$\mathrm{ABC}$ 分类管理法是意人利经济学家维尔弗雷多 - 帕累托于 19 世纪创立的一种管理方法, 它将存货按重 要程度分为 $\mathrm{ABC}$ 三类, 并根据其重要程度采用对应的管理方式 ${ }^{[1]}$ 。虽然 $\mathrm{ABC}$ 管理方法创立之初的目的是为 提高存货管理水平和资金使用效率，但实际使用效果表明，它同样适用于人力资源管理，成为激发不同类 型员工的积极性和主动性, 实现组织目标的重要方法。

新建本科院校是在 20 世纪 90 年代后期以来, 主要由原专科院校升格组建而成, 经过 20 多年的发展, 已成长为我国高等教育重要组成部分。图书馆作为新建本科高校发展的支柱之一, 推行职工 $\mathrm{ABC}$ 管理, 对 于充分发挥职工的主观能动性, 推动图书馆建设, 服务教学科研具有重要的意义和作用。

\section{1 新建本科院校图书馆职工现状}

新建本科院校图书馆职工可分为三类, 第一类是 $\mathrm{A}$ 类职工, 他们构成图书馆的核心队伍, 决定图书馆 的发展方向; 第二类是 B 类职工, 他们构成图书馆的支撑队伍, 对图书馆整体业务的推进和实施具有重要 影响; 第三类是 C 类职工, 他们构成图书馆的基础队伍, 承担着库室管理等业务工作 ${ }^{[2]}$ 。 
表 1 新建本科院校图书馆 $A B C$ 类职工结构分析表

\begin{tabular}{|c|c|c|c|}
\hline 项目 & A 类职工 & $\mathrm{B}$ 类职工 & $\mathrm{C}$ 类职工 \\
\hline 在工作中的地位 & 决定性影响 & 有一定影响 & 影响小或无影响 \\
\hline 学历、职称情况 ${ }^{[3]}$ & $\begin{array}{c}\text { 硕士及以上学历或副高及 } \\
\text { 以上职称 }\end{array}$ & 本科学历或中级职称 & $\begin{array}{c}\text { 专科及以下学历, 初 } \\
\text { 级职称或无职称 }\end{array}$ \\
\hline 专业背景 ${ }^{[4]}$ & $\begin{array}{c}\text { 受过图情专业教育, 或较为 } \\
\text { 系统的图情专业在职培训 }\end{array}$ & $\begin{array}{c}\text { 受过较为系统的图情专 } \\
\text { 业在职培训 }\end{array}$ & $\begin{array}{c}\text { 基本没有接受过图情 } \\
\text { 专业教育或培训 }\end{array}$ \\
\hline 职业性质 ${ }^{[5]}$ & 专业馆员 & 专业馆员或辅助馆员 & 辅助馆员 \\
\hline 工作特点 & $\begin{array}{c}\text { 强大的责任心、事业心、创 } \\
\text { 新精神和主动工作意识, 职 } \\
\text { 业发展方向明确 }\end{array}$ & $\begin{array}{c}\text { 有一定的责任心、事业 } \\
\text { 心和创新精神, 职业发 } \\
\text { 展不明确 }\end{array}$ & $\begin{array}{l}\text { 缺乏责任、事业心、 } \\
\text { 创新意识和职业发展 } \\
\text { 方向, 工作得过且过 }\end{array}$ \\
\hline 所从事岗位特点 & 核心岗位 & 重点岗位或普通岗位 & 普通岗位 \\
\hline 工作业务含量 & 高 & 中 & 低 \\
\hline
\end{tabular}

在企业人力资源管理中，认为 A、B、C 类职工分别占职工总数的 $20 \% 、 70 \%$ 和 $10 \%{ }^{[6]}$ 。对于新建本科院 校图书馆, 其 A、B、C 类职工比例结构与企业相比, A 类职工比例低于企业, C 类职工比例高于企业, $A B C$ 类职工结构不合理。造成这种情况的主要原因在于: 第一, 缺乏有效的人才积累。新建本科院校图书馆主 要来源于原专科院校图书馆, 基于专科办馆的职工结构不足以满足本科办馆需求, 缺乏本科办馆所需的 $\mathrm{A}$ 类职工和优秀 $\mathrm{B}$ 类职工的积累。第二，人才引进困难。在新建本科院校完成从专科教育模式向本科教育模 式的转型过程中, 学校重点关注专业教师队伍建设, 图书馆难以获得应有的关注; 同时由于高水平人才稀 缺, 造成图书馆, 尤其是非一线城市的图书馆对高水平人才缺乏吸引力。第三, 图书馆依然还承担着学校 引进人才家属等人员的安置职能 ${ }^{[7]}$ 。

\section{2 新建本科院校图书馆开展职工 $\mathrm{ABC}$ 管理的必要性}

\section{1 提升职工素质的需求}

引入职工 $\mathrm{ABC}$ 管理制度，能明确职工等级及其在图书馆工作的作用，充分实现职工的分层管理，全面 调动图书馆职工的工作热情, 促使他们全身心地投入到工作中具有重要作用。同时还能准确掌握不同等级 职工的特点和工作需求, 制定职工培训工作计划, 使得 $\mathrm{ABC}$ 类职工通过参加针对性的业务培训, 切实提升 业务素质和业务技能，确保图书馆业务工作能获得强有力的人力资源支持。

\section{2 完善图书馆职工等级建设和转换}

职工 $\mathrm{ABC}$ 管理的实施，能促使新建本科院校图书馆完成基于职工 $\mathrm{ABC}$ 管理需要的分类标准体系建设, 为确定职工等级建设提供依据; 完成 $\mathrm{C}$ 类职工向 $\mathrm{B}$ 类职工和 $\mathrm{B}$ 类职工向 $\mathrm{A}$ 类职工晋升的通道, 为职工等级 转换指引方向。职工在充分掌握等级划分标准的基础上, 明确自己等级, 同时也能在了解自己不足的基础 上, 制定发展方向, 实现由低级别向高级别转换。因此说, 职工 ABC 管理能有效实现职工的良性循环, 为 图书馆的可持续发展提供有效的后备力量支持。 


\section{3 有助于实现职工配置的科学化}

实现职工一岗位的科学匹配, 使每一个职工都能获得与其能力、职业发展等相匹配的岗位是新建本科 院校图书馆实现其发展目标的基础 ${ }^{[8]}$ 。传统的职工一岗位匹配主要依赖管理人员的经验与主观判断, 在开展 职工一岗位匹配工作中，更容易用性别、年龄、职称等依附于人身的客观物质差异为标准，忽视了内化在 人身中的素质能力的重要作用, 极易出现职工一岗位不匹配的情况。职工 $\mathrm{ABC}$ 管理是在对职工素质进行准 确评测和岗位要求等进行全面描述的基础上, 在充分考虑内化于职工人身中的业务素质的基础上, 开展职 工-岗位匹配工作, 有力地提升了职工一岗位匹配的公开化与科学化水平, 打破传统的论资排辈等对职工岗位配置的负面影响, 实现职工素质和岗位要求之间的高度一致, 有效消除传统的职工-岗位配置中的弊 端, 实现职工一岗位配置的最优化的目标。

\section{4 有助于进一步优化素质评测}

素质评测作为职工管理核心环节，切实提升素质评测的科学性、准确性，是提高职工管理水平的关 键因素。职工 $\mathrm{ABC}$ 管理中所确定的等级指标体系, 能根据不同等级的职工, 采取不同的指标体系进行评价, 实现职工素质的分层素质评价, 不但有效提高了职工素质评测的科学性, 为职工管理提供有效的素质评测 保障 ${ }^{[9]}$ 。通过素质评测, 可以及时掌握职工各方面变化的信息, 为调整职工-岗位匹配提供依据, 实现职工 管理由静态管理向动态管理的转变, 进一步提高职工管理的科学化、合理化水平 ${ }^{[10]}$ 。

\section{3 新建本科院校图书馆职工 $\mathrm{ABC}$ 管理方法}

\section{$3.1 \mathrm{~A}$ 类职工管理方法: 授权式管理}

$\mathrm{A}$ 类职工具有良好的业务素质和强烈的事业心、责任心及自主创新意识, 能主动根据图书馆发展需要 确定工作目标，对他们宜采用授权式管理。在图书馆审核的基础上，为其提供人、财、物保障，赋予其自 主决策、行动的权力, 为其工作的开展提供一个相对宽松的环境, 图书馆主要起监督保障的作用。

$\mathrm{A}$ 类职工具有扎实的专业知识、较高的业务素质和工作经验, 对他们的培训应着眼于创新意识与创新 能力的提升, 主要以参加海外或国内举办的、有关图书馆发展前沿动态为主要内容的高水平培训。

\subsection{B 类职工的管理: 参与式管理}

$B$ 类职工业务素质或完成工作的主观意愿上存在缺陷, 责任心、事业心和创新精神都有待提高, 但他 们又是图书馆业务工作最主要的执行者。为了充分调动 $\mathrm{B}$ 类职工的主观能动性, 对其宜采用参与式管理。 首先图书馆要明确 $\mathrm{B}$ 类职工的工作内容和要求, 使其能准确了解、掌握所承担的工作。其次图书馆应支持 他们按自己的想法开展工作, 在其充分履职的基础上, 尽量减少对其工作的指示和约束。再次要建立起有 效的沟通机制, 主动加强与 $\mathrm{B}$ 类职工的沟通与交流, 让他们参与决策, 激发他们的创新意识。最后要协助 他们规划职业生涯, 强化他们对自我和图书馆的认知, 增强他们在职业生涯规划中的主动性, 确保他们的 职业规划与图书馆发展目标保持高度一致，促使他们能尽快成长与成熟。

B 类职工是图书馆业务工作开展的支撑力量, 具有一定的专业知识和创新能力, 因此对他们的培训应 同时兼顾图书馆专业知识和发展前沿动态。通过图书馆专业知识培训, 有效弥补其专业知识的不足, 为其 业务工作的完成构建良好的专业基础; 通过参加图书馆发展前沿发展动态的培训, 使其了解图书馆发展趋 势, 开阔工作思路, 提高创新意识, 促使他们向 A 类职工晋升。 


\section{3 C 类职工的管理: 指示式管理}

因 C 类职工的业务素质存在较大不足, 且工作的主动性较为欠缺, 因此对他们宜采用指示式管理。图 书馆主要采取单向沟通形式, 明示 C 类职工做什么、怎么做、如何做、何时做, 并通过完善的管理规定或 业务细则等将其工作内容、流程、要求等固化下来, 规范其行为, 确保其能按要求完成所承担的工作。

$C$ 类职工业务知识薄弱, 难以满足业务工作需要, 因此对 $\mathrm{C}$ 类职工应以基础业务知识培训为重点, 逐 步引入 $\mathrm{B}$ 类职工培训内容，使其在完成所承担工作的同时向 $\mathrm{B}$ 类职工晋升。

\section{4 新建本科院校图书馆职工 $\mathrm{ABC}$ 管理实施步骤}

新建本科院校图书馆实施职工 $\mathrm{ABC}$ 管理, 通常可分为前期准备、等级确定和管理实施三个阶段。

\section{1 前期准备阶段}

主要完成制度体系建设和等级标准制定。根据职工 $\mathrm{ABC}$ 管理的需要, 构建包含管理原则、管理方法、 考评制度、奖惩规定、等级调整等内容的职工 $\mathrm{ABC}$ 管理规章制度体系, 为职工 $\mathrm{ABC}$ 管理的开展提供有效的 制度保障。同时以职工学历、职称、专业背景教育、工作经验、岗位性质等为依据, 制定等级分类指标体 系, 为科学确定职工等级提供依据。为确保指标体系的科学性, 在构建指标体系时要坚持唯一性原则和完 备性原则。唯一性原则是指每一个指标所反应的内容必须是唯一的，不同指标的内涵不能有交叉融合的现 象。完备性原则是指标体系应覆盖确定职工等级的全部参数, 不能有所遗漏。

\section{2 等级确定阶段}

主要完成职工信息搜集评价和职工等级确定工作。根据等级分类指标体系, 完成与等级确定有关的职 工信息搜集、整理等工作, 并通过选拔性评测、配置性评测、开发性评测、和诊断性评测等方法确定职工 的等级。其中选拔性评测重点是要遴选出优秀职工并给予对应的等级, 配置性评测主要是通过评测结果确 定等级, 开发性评测是选拔具有培养价值并能在较短时间内从现有等级晋升到上一等级的职工, 诊断性评 测则是指找出有可能产生负面影响的职工，确定其等级和管理方法，避免负面效应的出现。

\section{3 管理实施阶段}

主要完成职工 $A B C$ 管理实施和等级调整工作。根据职工 $\mathrm{ABC}$ 管理需要，全面推行职工 $\mathrm{ABC}$ 管理，确保 职工 $\mathrm{ABC}$ 管理得到有效的贯彻落实。通过考评工作的开展, 对职工履行 $\mathrm{ABC}$ 管理的情况进行评判, 落实奖 惩规定, 对于满足晋升上一级等级的职工应及时晋升, 对于未能完成要求的职工则应及时将其调整到下一 等级。

\section{5 结语}

本文在对新建本科院校图书馆职工现状进行全面分析的基础上，阐述了新建本科院校图书馆实施职工 $\mathrm{ABC}$ 管理, 对于提高其职工整体素质, 解决其建设与发展中人力资源不足具有重要的意义和作用。指出新 建本科院校图书馆结合自身条件, 推行职工 $\mathrm{ABC}$ 管理已成为其不得不考虑的重要问题。

\section{6 致谢}

感谢住房和城乡建设部 (项目编号：2015-K8-006) 和江苏高校哲学社会科学研究 (项目编号: 2012SJD870010) 基金的资助。

This work was supported by Ministry of Housing and Urban-Rural Development of the People's Republic of China (2015-K8-006) and universities' philosophy and social science researches in Jiangsu 
Provinc(2012SJD870010).

\section{参考文献:}

[1] 廖丽平．基于库存管理理论的人力资源价值管理. [J] 广东水利电力职业技术学院学报，2010（1)：38-42

[2] 吴敏. SECC 公司 ABC 建立项目人力资源管理研究 [D]．成都：电子科技大学，2014

[3] [4] [5] 教育部. C (教高【2015】14 号)

[6] 杨玲. 新建本科院校图书馆人力资源管理探析：以安徽省为例 $[J]$. 湖北科技学院学报，2016（5）:117-120

$[7]$ 杨淑玲．高校图书馆人力资源开发与管理 $[J]$ 。 内蒙古民族大学学报，2005（1）：115-118

[8］崔文媛．高校图书馆创新的人力资源管理 [J]．现代情报，2007（12）：114-116

[9] 刘睿．人力资源经理绩效考核指标体系的构建 $[J]$. 企业经济，2012（1)：81-84

[10］洪涛. 人力资源管理对员工工作绩效的影响观察 [J]．人力资源管理，2015(12) :21-22

\section{References:}

[1] Liao Liping. Human Resource Value Management Based on Inventory Management Theory [J]. Journal of Guangdong Technical College of Water Resources and Electric Engineering, 2010(1):38-42

[2] Wu Min. Research on Human Resource Management of ABC Supervision Project in SECC Company [D].Chengdu: University of Electronic Science and Technology of China, 2014 [3] [4] [5] Ministry of Education. C (JG [2015] No. 14)

[6] Yang Ling. Analysis on the Human Resource Management of the Newly-built University Library: Taking Anhui Province as an Example [J]. Journal of Hubei University of Science and Technology,2016(5):117-120

[7] Yang Shu-ling. Development and Management of Human Resources in the University Library [J]. Journal of Inner Mongolia University for Nationalities, 2005(1):115-118

[8] Cui Wenyuan. Innovative Human Resource Management of University Library [J]. Journal of Modern Information,2007(12):114-116

[9] Liu Rui. Construction of Performance Evaluating System for Human Resource Managers [J].Enterprise Economy, 2012(1):81-84

[10] Hong Tao. Effect of Human Resource Management on Employee's Job Performance [J]. Human Resource Management, 2015(12):21-22 